\title{
Characterization of convex functions
}

\author{
by \\ JACEK TABOR (Kraków) and Józef TABor (Rzeszów)
}

\begin{abstract}
There are many inequalities which in the class of continuous functions are equivalent to convexity (for example the Jensen inequality and the Hermite-Hadamard inequalities). We show that this is not a coincidence: every nontrivial linear inequality which is valid for all convex functions is valid only for convex functions.
\end{abstract}

1. Introduction. There are many inequalities valid for convex functions. Probably the most well-known ones are the Jensen inequality

$$
f\left(\frac{x+y}{2}\right) \leq \frac{f(x)+f(y)}{2} \quad \text { for } x, y \in \mathbb{R},
$$

and the Hermite-Hadamard inequalities

$$
f\left(\frac{x+y}{2}\right) \leq \frac{1}{y-x} \int_{x}^{y} f(z) d z \leq \frac{f(x)+f(y)}{2} \quad \text { for } x, y \in \mathbb{R}, x<y .
$$

In fact, in the class of continuous functions, each of the above inequalities is equivalent to convexity (see [NP, Chapter 1]; the same concerns Popoviciu's inequality [NP, Th. 1.18]).

It is usually easy to check whether a given linear inequality holds for all convex functions with domain in $\mathbb{R}$. Namely, it is enough to verify that inequality for the functions $x \mapsto|x-p|$ for all $p \in \mathbb{R}$ (see [NP, comments after Theorem 1.5.7]). As a consequence one can prove an even more widely applicable result, which is an easy corollary of Popoviciu's Theorem [NP, Th. 4.2.7].

Popoviciu's Theorem. Let $\nu, \mu$ be finite positive Borel measures on $[a, b]$. Then

$$
\int_{a}^{b} f(x) d \nu(x) \leq \int_{a}^{b} f(x) d \mu(x)
$$

2000 Mathematics Subject Classification: 26B25, 39B62.

Key words and phrases: convex function, Jensen inequality, approximation. 
for all continuous convex functions $f:[a, b] \rightarrow \mathbb{R}$, if and only if $\nu([a, b])=$ $\mu([a, b])$ and

$$
\begin{aligned}
& \int_{a}^{t}(t-x) d \nu(x) \leq \int_{a}^{t} d \mu(x) \\
& \int_{t}^{b}(x-t) d \nu(x) \leq \int_{t}^{b}(x-t) d \mu(x) \quad \text { for } t \in[a, b] .
\end{aligned}
$$

In this paper we deal with a problem, to some extent, opposite. Namely, we prove that every nontrivial (linear-type) inequality which is valid for all convex functions, gives in fact a characterization of convexity in the class of continuous functions. In particular, as a direct consequence of Theorem 2 below we obtain the following result:

TheOrem. Let $K$ be a compact subset of $\mathbb{R}^{n}$ and let $\nu$ and $\mu$ be distinct finite Borel measures on $K$. Assume that

$$
\int_{K} f(x) d \nu(x) \leq \int_{K} f(x) d \mu(x)
$$

for every continuous convex real-valued function $f$ such that $K \subset \operatorname{dom}(f)$ (where dom denotes domain). Let $W$ be a convex subset of a Banach space $E$ and let $h \in C(W, \mathbb{R})$ be such that

$$
\int_{K} h(a(x)) d \nu(x) \leq \int_{K} h(a(x)) d \mu(x)
$$

for every affine function $a: \mathbb{R}^{n} \rightarrow E$ such that $a(K) \subset W$. Then $h$ is convex.

For more information on convex functions we refer the reader to $[\mathrm{Ku}$, NP, Ro].

2. Approximation. Let $K$ be a compact convex subset of $\mathbb{R}^{n}$. We denote by $C(K, \mathbb{R})$ the Banach space of all continuous functions from $K$ into $\mathbb{R}$ with the supremum norm. For a Lipschitz function $g \in C(K, \mathbb{R})$, we denote by $\operatorname{lip}(g)$ the smallest Lipschitz constant of $g$. Let $\operatorname{Aff}(K)$ denote the set of all affine functions from $K$ into $K$, and $\operatorname{Aff}_{\varepsilon}(K)$ the subset of $\operatorname{Aff}(K)$ consisting of functions with Lipschitz constant less than or equal to $\varepsilon$. Given a set $B \subset C(K, \mathbb{R})$, we denote by wedge $(B)$ the smallest wedge containing $B$, where by wedge we understand a closed convex and positively homogeneous set.

For $f \in C(K, \mathbb{R})$, we say that $f \in C^{k}$ if there exists an open neighbourhood $U$ of $K$ and $f_{U} \in C^{k}(U, \mathbb{R})$ such that $\left.f_{U}\right|_{K}=f$.

Let $\operatorname{Conv}(K) \subset C(K, \mathbb{R})$ be the set of all convex functions on $K$. 
Given $g \in C(K, \mathbb{R})$ and $\varepsilon>0$ we put

$$
\operatorname{Aff}_{\varepsilon}(g):=\left\{g \circ a: a \in \operatorname{Aff}_{\varepsilon}(K)\right\} .
$$

Our aim in this section is to show that an arbitrary function from $C(K, \mathbb{R})$ can be approximated in the supremum norm by a sum (with nonnegative coefficients) of a convex function and affine modifications of a fixed nonconvex one.

Theorem 1. Let $K \subset \mathbb{R}^{n}$ be a compact convex set and let $g: K \rightarrow \mathbb{R}$ be a continuous function which is not convex. Let $\varepsilon>0$. Then

$$
C(K, \mathbb{R})=\operatorname{wedge}\left(\operatorname{Conv}(K) \cup \operatorname{Aff}_{\varepsilon}(g)\right) .
$$

We postpone the proof to the end of this section and precede it by a few auxiliary lemmas. We use the following notation. Let $\eta$ be a mollifier, that is, a nonnegative function from $C^{\infty}\left(\mathbb{R}^{n}, \mathbb{R}\right)$ with support in the unit ball $B(0,1)$ and such that $\int \eta=1$. For $\delta>0$ we put $\eta_{\delta}(x):=\eta(x / \delta) / \delta^{n}$.

Given a function $f$ defined on a subset of $\mathbb{R}^{n}$ and $x \in \mathbb{R}^{n}$, let $T_{x} f$ the function $T_{x} f: y \mapsto f(y-x)$. Given $\varepsilon>0$ we define the homothety at $x$ by

$$
H_{x}^{\varepsilon}(y):=x+\varepsilon(y-x) \quad \text { for } y \in \mathbb{R}^{n} .
$$

Lemma 1. Let $W$ be a convex compact subset of $\mathbb{R}^{n}$ with nonempty interior, let $r>0$ and let $g \in C(W+B(0, r), \mathbb{R})$. For $\delta \in(0, r)$ define $g_{\delta}: W \rightarrow \mathbb{R}$ by the formula

$$
g_{\delta}(x):=\int_{B(0, \delta)} \eta_{\delta}(y) g(x-y) d y \quad \text { for } x \in W .
$$

Then

(i) $g_{\delta} \in C^{\infty}(W, \mathbb{R})$;

(ii) $\lim _{\delta \rightarrow 0^{+}} g_{\delta}=\left.g\right|_{W}$ in $C(W, \mathbb{R})$;

(iii) $g_{\delta} \in$ wedge $\left\{\left.\left(T_{a} g\right)\right|_{W}: a \in B(0, \delta)\right\}$.

Proof. Since (i) and (ii) are well-known (see for example [Ev, Appendix C.4]), we sketch the proof of (iii). Given $\varepsilon>0$, by uniform continuity of $g$ on compact sets we find $\delta^{\prime}>0$ such that

$$
\left|g(x-y)-g\left(x-y^{\prime}\right)\right| \leq \varepsilon \quad \text { whenever } x \in W, y, y^{\prime} \in B(0, \delta),\left\|y-y^{\prime}\right\| \leq \delta^{\prime} .
$$

Decompose $B(0, \delta)$ into a disjoint union of finitely many measurable subsets $\left\{Y_{i}\right\}$ with diameter less than $\delta^{\prime}$. Choose points $y_{i} \in Y_{i}$ arbitrarily and put

$$
h:=\left.\sum_{i} \int_{Y_{i}} \eta_{\delta}(y) d y \cdot\left(T_{y_{i}} g\right)\right|_{W} .
$$

Clearly, $h \in$ wedge $\left\{\left.\left(T_{a} g\right)\right|_{W}: a \in B(0, \delta)\right\}$. We finish the proof by showing 
that $h$ approximates $g_{\delta}$ in the supremum norm. Indeed, for $x \in W$,

$$
\begin{aligned}
\left|g_{\delta}(x)-h(x)\right| & =\left|\sum_{i} \int_{Y_{i}} \eta_{\delta}(y) g(x-y) d y-\sum_{i} \int_{Y_{i}} \eta_{\delta}(y) g\left(x-y_{i}\right) d y\right| \\
& \leq \sum_{i} \int_{Y_{i}} \eta_{\delta}(y)\left|g(x-y)-g\left(x-y_{i}\right)\right| d y \\
& \leq \varepsilon \sum_{i} \int_{Y_{i}} \eta_{\delta}(y) d y=\varepsilon \int_{B(0, \delta)} \eta_{\delta}(y) d y=\varepsilon . \mathbf{}
\end{aligned}
$$

Lemma 2. Let $K$ be a compact convex subset of $\mathbb{R}^{n}$ with nonempty interior and let $a \in K$ and $r>0$ be such that $B(a, r) \subset K$. Let $\varepsilon \in(0,1)$ and $g \in C(K, \mathbb{R})$. Set

$$
\bar{g}:=g \circ H_{a}^{\varepsilon} .
$$

Then

$$
\left.\left(T_{x} \bar{g}\right)\right|_{K} \in \operatorname{Aff}_{\varepsilon}(g) \quad \text { for } x \in B(0,(1-\varepsilon) r / \varepsilon) .
$$

Proof. By the convexity of $K$,

$$
H_{b}^{\varepsilon}(K) \subset K \quad \text { for } b \in K .
$$

Pick $x \in B(0,(1-\varepsilon) r / \varepsilon)$. Then $\|\varepsilon x /(1-\varepsilon)\|<r$ and so

$$
a+\frac{\varepsilon}{1-\varepsilon} x \in K \text {. }
$$

Hence $g \circ H_{a+\varepsilon x /(1-\varepsilon)}$ is well-defined and the equality

$$
T_{x} \bar{g}=g \circ H_{a+\varepsilon x /(1-\varepsilon)}^{\varepsilon}
$$

completes the proof.

LEMma 3. Let $K$ be a compact convex subset of $\mathbb{R}^{n}$ with nonempty interior. Let $\varepsilon \in(0,1)$ and suppose $g \in C(K, \mathbb{R})$ is not convex. Then there exists a $C^{\infty}$ function $h \in$ wedge $\left(\operatorname{Aff}_{\varepsilon}(g)\right)$ and $a \in \operatorname{int} K$ such that the function $K \ni x \mapsto D_{a}^{2} h[x]$ attains a negative value.

Proof. There exists a point $\bar{a} \in \operatorname{int} K$ such that $g$ is not convex on any open convex neighbourhood of $\bar{a}$. Let $r>0$ be such that $B(\bar{a}, r) \subset K$. We define $\bar{g}$ by the formula

$$
\bar{g}:=g \circ H_{\bar{a}}^{\varepsilon} .
$$

Since $H_{\bar{a}}^{\varepsilon}(B(\bar{a}, r)) \subset B(\bar{a}, r),\left.\bar{g}\right|_{K}$ is not convex. By a similar reasoning to that in the proof of Lemma 2 one can show that $K+B(0,(1-\varepsilon) r / \varepsilon) \subset$ $\operatorname{dom}(\bar{g})$. In virtue of Lemma 2 we have

$$
\left.\left(T_{x} \bar{g}\right)\right|_{K} \in \operatorname{Aff}_{\varepsilon}(g) \quad \text { for } x \in B(0,(1-\varepsilon) r / \varepsilon) .
$$

Making use of Lemma 1(iii) we obtain

$$
\left.\bar{g}_{\delta}\right|_{K} \in \operatorname{wedge}\left(\operatorname{Aff}_{\varepsilon}(g)\right)
$$

for sufficiently small $\delta$. 
By Lemma 1(ii) we know that $\lim _{\delta \rightarrow 0^{+}}\left\|\left.\bar{g}_{\delta}\right|_{K}-\left.\bar{g}\right|_{K}\right\|_{\text {sup }}=0$. Hence $h:=$ $\left.\bar{g}_{\delta}\right|_{K}$ is not convex for some small $\delta$. Since $h$ is a $C^{\infty}$ function, there exists an $a \in \operatorname{int} K$ such that the mapping $K \ni x \mapsto D_{a}^{2} h[x]$ is not nonnegative.

Now we are ready to prove the main result of this section.

Proof of Theorem 1. Without loss of generality we may assume that $\varepsilon<1$. Since every convex set has nonempty interior in the affine space spanned by it, it is enough to consider the case when int $K \neq \emptyset$. We put

$$
\mathcal{F}:=\operatorname{wedge}\left(\operatorname{Conv}(K) \cup \operatorname{Aff}_{\varepsilon}(g)\right) .
$$

We are going to show that

$$
f \circ a \in \mathcal{F} \quad \text { for } f \in \mathcal{F}, a \in \operatorname{Aff}(K), \operatorname{lip}(a) \leq 1 .
$$

This follows from the fact that the above inclusion trivially holds for $f \in$ $\operatorname{Conv}(K)$ and $f \in \operatorname{Aff}_{\varepsilon}(g)$, and consequently also for functions belonging to the wedge spanned over $\operatorname{Conv}(K) \cup \operatorname{Aff}_{\varepsilon}(g)$. Let $\mathcal{G}$ denote the set of functions of the form

$$
f+\sum_{i=1}^{n} \alpha_{i} f_{i},
$$

where $f \in C(K, \mathbb{R})$ is convex, $f_{i} \in \operatorname{Aff}_{\varepsilon}(g), \alpha_{i} \geq 0$ and $n \in \mathbb{N}$. As one can easily check, a version of (1) holds for $\mathcal{G}$, and consequently also for $\mathcal{F}=\operatorname{cl} \mathcal{G}$.

By Lemma 3 there exists a function $h \in \mathcal{F}$ of class $C^{\infty}$ and $p \in \operatorname{int} K$ such that the mapping $A:=x \mapsto D_{p}^{2} h[x]$ is not nonnegative. Clearly without loss of generality (we can shift the origin of the coordinate system to $p$ ) we may assume that $p=0$. Then $0 \in \operatorname{int} K$.

Now the function $\bar{h}: K \ni x \mapsto h(x)-h(0)-D_{0} h[x]$ is also an element of $\mathcal{F}$, as $\bar{h}-h$ is affine. We have

$$
\bar{h}(x)=D_{0}^{2} h[x]+o\left(\|x\|^{2}\right)=A(x)+o\left(\|x\|^{2}\right) \quad \text { for } x \in K .
$$

For $M \geq 1$ we define the function $h_{M}: K \rightarrow \mathbb{R}$ by the formula

$$
h_{M}(x):=M^{2} \bar{h}(x / M) \quad \text { for } x \in K .
$$

Since $0 \in K$ and $K$ is convex, $h_{M}$ is well-defined. Since we have $h_{M}(x)=$ $M^{2}\left(\bar{h} \circ H_{0}^{1 / M}\right)(x)$ and $\operatorname{lip}\left(H_{0}^{1 / M}\right)=1 / M$, by (1) we see that $h_{M} \in \mathcal{F}$. As $h_{M}$ tends uniformly (as $M \rightarrow \infty$ ) to $A$, we conclude that $A \in \mathcal{F}$.

Because $A$ attains a negative value, there exists an orthonormal basis $e_{1}, \ldots, e_{n}$ of $\mathbb{R}^{n}$ such that $A\left(e_{1}\right)<0$. From now on we change the canonical base to the new one. Then for $\lambda:=A\left(e_{1}\right)$ we have

$$
A\left(x_{1}, 0, \ldots, 0\right)=\lambda x_{1}^{2} .
$$

Consider the map

$$
P_{k}\left(x_{1}, \ldots, x_{n}\right)=\left(x_{k}, 0, \ldots, 0\right) \quad \text { for } k=1, \ldots, n .
$$


Since $0 \in \operatorname{int} K$ and $K$ is bounded, there exists $\delta \in(0,1]$ such that

$$
\left.\delta \cdot P_{k}\right|_{K} \in \operatorname{Aff}_{\varepsilon}(K) \quad \text { for } k=1, \ldots, n .
$$

Consequently, by (1), $p_{k}:=A \circ\left(\left.\delta \cdot P_{k}\right|_{K}\right) \in \mathcal{F}$. Then

$$
p_{k}\left(x_{1}, \ldots, x_{n}\right)=\lambda \delta^{2} x_{k}^{2} \quad \text { for }\left(x_{1}, \ldots, x_{n}\right) \in K \text {. }
$$

Since $\lambda<0$, we see that the function $P_{k}: K \in x=\left(x_{1}, \ldots, x_{n}\right) \mapsto-x_{k}^{2}$ is also an element of $\mathcal{F}$. Consequently, the function

$$
x=\left(x_{1}, \ldots, x_{n}\right) \mapsto-\left(x_{1}^{2}+\cdots+x_{n}^{2}\right)=-\|x\|^{2}
$$

is an element of $\mathcal{F}$.

Now we are ready to show that $C(K, \mathbb{R}) \subset \mathcal{F}$. First consider the case of $C^{\infty}$ functions. Let $f$ be a $C^{\infty}$ function on $K$ (that is, a restriction to $K$ of a $C^{\infty}$ function on the neighbourhood of $K$ ). Clearly, there exists $M>0$ such that the function

$$
F: K \ni x \mapsto f(x)+M\|x\|^{2}
$$

is convex, which implies that $F \in \mathcal{F}$. Since the function $K \ni x \mapsto-M\|x\|^{2}$ is also an element of $\mathcal{F}$, we deduce that

$$
K \ni x \mapsto F(x)+\left(-M \cdot\|x\|^{2}\right)=f(x)
$$

is also an element of $\mathcal{F}$.

By Lemma 1, the class of $C^{\infty}$ functions is dense in $C(K, \mathbb{R})$. This completes the proof of Theorem 1 .

REMARK 1. The assertion of Theorem 1 can be reformulated in the following way. Every continuous function $h: K \rightarrow \mathbb{R}$ can be uniformly approximated by functions of the form

$$
f+\sum_{i=1}^{n} \alpha_{i} f_{i},
$$

where $f$ is a continuous convex function, $f_{i} \in \operatorname{Aff}_{\varepsilon}(g), \alpha_{i} \geq 0$ and $n \in \mathbb{N}$.

3. Convex inequalities. Now we are ready to proceed to our main subject of interest, that is, to inequalities valid for convex functions.

TheOREM 2. Let $K$ be a compact convex subset of $\mathbb{R}^{n}$. Let $\nu$ and $\mu$ be distinct finite positive Borel measures in $K$. Assume that

$$
\int_{K} f d \nu \leq \int_{K} f d \mu
$$

for every continuous convex function $f: K \rightarrow \mathbb{R}$. Let $E$ be a Banach space. Let $\varepsilon>0$. Let $W \subset E$ be a convex set and let $h \in C(W, \mathbb{R})$ be such that

$$
\int_{K}(h \circ a) d \nu \leq \int_{K}(h \circ a) d \mu
$$


for every one-dimensional affine function $a: \mathbb{R}^{n} \rightarrow E$ such that $a(K) \subset W$ and $\operatorname{lip}(a)<\varepsilon$. Then $h$ is convex.

Proof. Suppose that $h$ is not convex. Then there exist $w_{0}, w_{1} \in W$ such that $h$ is not convex on the interval $\left[w_{0}, w_{1}\right]$. We define $\bar{h}:[0,1] \rightarrow \mathbb{R}$ by

$$
\bar{h}(t)=h\left(w_{0}+t\left(w_{1}-w_{0}\right)\right) \quad \text { for } t \in[0,1] .
$$

Obviously $\bar{h}$ is a continuous function which is not convex, and therefore we can find $0<t_{1}<t_{2}<1$ such that $\left.\bar{h}\right|_{\left[t_{1}, t_{2}\right]}$ is not convex. Let

$$
\bar{t}:=\inf \left\{t \in\left[t_{1}, t_{2}\right]:\left.\bar{h}\right|_{\left[t_{1}, t\right]} \text { is not convex }\right\} .
$$

Since $\left.h\right|_{\left[t_{1}, \bar{t}\right]}$ is convex and $\left.h\right|_{\left[t_{1}, t\right]}$ is not convex for any $t>\bar{t}$, it follows that $\bar{h}$ is not convex on a neighbourhood of $\bar{t}$.

Now we choose an affine map $i: K \rightarrow[0,1]$ with $\bar{t} \in \operatorname{int}(i(K))$ and $\operatorname{lip}(i) \leq 1 /\left\|w_{1}-w_{0}\right\|$ and define

$$
a_{0}(x):=w_{0}+i(x)\left(w_{1}-w_{0}\right) \quad \text { for } x \in K .
$$

Obviously $\operatorname{lip}\left(a_{0}\right) \leq 1$. Let

$$
\mathcal{G}:=\left\{f \in C(K, \mathbb{R}): \int_{K} f d \nu \leq \int_{K} f d \mu\right\} .
$$

Obviously $\mathcal{G}$ is a wedge which contains all convex functions.

We put

$$
g:=h \circ a_{0} \in C(K, \mathbb{R}) .
$$

Clearly $g$ is not convex. Let $a: K \rightarrow K$ be an affine function with $\operatorname{lip}(a) \leq \varepsilon$. Then

$$
g \circ a=h \circ a_{0} \circ a .
$$

As $a_{0} \circ a: K \rightarrow W$ is affine with $\operatorname{lip}\left(a_{0} \circ a\right) \leq \varepsilon$, by (2) we see that $g \circ a \in \mathcal{G}$. This means that $\operatorname{Aff}_{\varepsilon}(g) \subset \mathcal{G}$.

Now Theorem 1 shows that $\mathcal{G}=C(K, \mathbb{R})$, and consequently

$$
\int_{K} f d \nu \leq \int_{K} f d \mu
$$

for every $f \in C(K, \mathbb{R})$. Putting $-f$ in place of $f$ we obtain

$$
\int_{K} f d \nu=\int_{K} f d \mu \quad \text { for } f \in C(K, \mathbb{R}),
$$

which trivially implies that the measures $\nu$ and $\mu$ are equal.

As a trivial consequence, the Jensen inequality and the Hermite-Hadamard inequalities in the class of continuous functions imply convexity. We provide the proof for the Hermite inequality in $\mathbb{R}$ (other proofs are similar).

Let $\delta_{a}$ denote the unit atom measure concentrated at $a$. 
COROllary 1. Let $W$ be a convex subset of a Banach space $E$ and let $g: W \rightarrow \mathbb{R}$ be a continuous function such that

$$
g\left(\frac{x+y}{2}\right) \leq \int_{0}^{1} g(x+t(y-x)) d t \quad \text { for } x, y \in W .
$$

Then $g$ is convex.

Proof. Let

$$
K=[0,1], \quad \nu=\delta_{1 / 2}, \quad \mu=\left.\lambda_{1}\right|_{K},
$$

where $\left.\lambda_{1}\right|_{K}$ denotes the one-dimensional Lebesgue measure restricted to $K$. Obviously $\nu \neq \mu$.

By the Hermite inequality for every convex function $f: K=[0,1] \rightarrow \mathbb{R}$ we obtain

$$
\int_{K} f d \nu=f(1 / 2) \leq \int_{0}^{1} f(s) d s=\int_{K} f d \mu .
$$

Now, by (3), for every affine function $a: K \rightarrow W$ such that $a(K) \subset W$ we have

$$
\begin{aligned}
\int_{K} g \circ a d \nu & =g(a(1 / 2)) \leq \int_{0}^{1} g(a(0)+t(a(1)-a(0))) d t \\
& =\int_{0}^{1} g(a(t)) d t=\int_{K} g \circ a d \mu .
\end{aligned}
$$

Consequently, by Theorem $2, g$ is convex.

In a similar manner one can prove that every continuous $t$-Wright convex function is convex. Recall that a function $f: V \rightarrow \mathbb{R}$, where $V$ is convex, is called $t$-Wright convex (where $t \in(0,1)$ ) if

$$
f(t x+(1-t) y)+f((1-t) x+t y) \leq f(x)+f(y) \quad \text { for all } x, y \in V .
$$

Then we take $\nu=\delta_{t_{0}}+\delta_{1-t_{0}}, \mu=\delta_{0}+\delta_{1}$.

The above mentioned result is well-known [MNP]. We present it to point out that Theorem 2 can be applied as a useful tool in the theory of convex functions.

REMARK 2. One can ask if the space of affine transformations in Theorem 2 can be replaced by a smaller one. We show that the space of affine similarities is not sufficient.

Consider the inequality

$$
f(0) \leq \frac{1}{2 \pi} \int_{S(0,1)} f(x) d S(x),
$$


which is clearly satisfied for all subharmonic (and consequently also convex) functions $f$ on $\mathbb{R}^{2}$.

Let $g\left(x_{1}, x_{2}\right)=x_{1}^{2}-x_{2}^{2}$. One can easily check that $g \circ a$ satisfies the above inequality (in fact even equality) for every affine similarity $a$ (this is because $g$ is harmonic). However, clearly $g$ is not convex.

Problem 1. Are Theorems 1 and 2 valid in infinite-dimensional Banach spaces?

Acknowledgements. We would like to thank the referee for valuable remarks.

\section{References}

[Ev] L. Evans, Partial Differential Equations, Amer. Math. Soc., 1998.

[Ku] M. Kuczma, An Introduction to the Theory of Functional Equations and Inequalities, PWN, 1985.

[MNP] Gy. Maksa, K. Nikodem and Zs. Páles, Results on t-Wright convexity, C. R. Math. Rep. Acad. Sci. Canada 13 (1991), 274-278.

[NP] C. Niculescu and L.-E. Persson, Convex Functions and Their Applications, CMS Books in Math. 23, Springer, 2006.

[Ro] R. Rockafellar, Convex Analysis, Princeton Math. Ser. 28, Princeton Univ. Press, 1970.

Jacek Tabor

Institute of Mathematics

Jagiellonian University

Łojasiewicza 6

30-348 Kraków, Poland

E-mail: jacek.tabor@im.uj.edu.pl
Józef Tabor

Institute of Mathematics University of Rzeszów Rejtana $16 \mathrm{~A}$

35-310 Rzeszów, Poland E-mail: tabor@univ.rzeszow.pl

Received May 10, 2007

Revised version December 19, 2008 